\title{
Integrated modelling and analysis of micro-cutting mechanics with the precision surface generation in abrasive flow machining
}

\author{
Yizhi Shao $^{1} \cdot$ Kai Cheng ${ }^{1}$
}

Received: 21 May 2018 / Accepted: 12 March 2019/Published online: 3 April 2019

(C) The Author(s) 2019

\begin{abstract}
Abrasive flow machining (AFM) technology is attracting more and more attention and keeps expanding into more areas by the industry and research community particularly in the context of increasing demands for post-processing of the complex aerofoil structures and additively manufactured components. It is fundamentally vital to develop an industrial feasible approach to controlling and improving the surface roughness of the structure and component, and even the profile accuracy and surface texture. In this paper, a multiscale multiphysics approach combining with micro-cutting mechanics is presented for modelling and analysis of the surface roughness and topography profile generation in the AFM process. The analysis is developed and implemented by using MATLAB programming integrated with the COMSOL multiphysics computational environment. Micro-cutting mechanics modelling and the Monte Carlo (MC) algorithms are integrated to develop simulations on the AFM generation of surface texture and topography through abrasive micro-machining with thousands of grains under complex multiscale and multiphysics working conditions. Well-designed AFM experiment trials on machining aerofoil structures are carried out to further evaluate and validate the modelling and analysis. The work presented is fundamental but essential as a part of the project for developing the simulation-based AFM virtual machining system.
\end{abstract}

Keywords Abrasive flow machining $\cdot$ Micro-cutting $\cdot$ Multiscale modelling $\cdot$ Monte Carlo algorithms $\cdot$ Multiphysics simulation $\cdot$ Surface roughness $\cdot$ Aerofoil structures

\begin{tabular}{ll}
\multicolumn{2}{l}{ Nomenclature } \\
$\bar{\sigma}$ & Contact area \\
$\nabla$ & Gradient \\
$\dot{\gamma}$ & Shear rate \\
$\rho$ & Density \\
$d^{\prime}$ & Depth of cut \\
$E_{m}$ & Young's modulus \\
$H_{W}$ & Hardness of material \\
AFM & Abrasive flow machining \\
CNC & Computer numerical control \\
CFD & Computational fluid dynamics \\
IBR & Integrally bladed rotor \\
MC & The Monte Carlo algorithms \\
MRR & Material removal rate \\
SR & Surface roughness
\end{tabular}

Kai Cheng

kai.cheng@brunel.ac.uk

1 Institute of Materials and Manufacturing, Brunel University London, Uxbridge UB8 3PH, UK

\section{Introduction}

Abrasive flow machining (AFM) was first brought into the manufacturing industry in the 1960s. AFM is a process where material removal (MR) and surface roughness (SR) improvement are attained by extruding a viscoelastic fluid carrying abrasive grits through a workpiece. It is normally used when interior features need to be polished, rounded or de-burred and are unreachable by conventional processes. Currently, in the aeronautic manufacturing industry, AFM is often performed by extruding high viscosity media, through workpieces featured with complex free-form aerostructures. AFM is also an essential means for surface post-processing particularly for additively manufactured 3D complex components. However, the AFM process still has a long way from being applied in a more predictable, producible and highly productive manner especially against the increasing demands on surface finish/ uniformity and profile edge accuracy control at the aerofoil structures and components. Furthermore, the optimisation and predictive control of AFM processes can help lead to the substantial improvement of aerofoil structures and components in their machining accuracy and thus their performance as well. 
Aircraft engines are high-technology products, which need innovative enabling manufacturing techniques for continuous improvement of the engines' fuel consumption and noise levels, and their enhanced reliability and safety, while simultaneously coping with ever stringent environmental legislations. An integrally bladed rotor (IBR) can be produced by CNC machining from a single forged part or by welding individual blades to a disc structure. IBR manufacture requires polishing the blades to the required surface roughness while maintaining tight accuracy control of the entire edge profile of the blades, i.e. particular attention being paid to the geometry of the leading edge and trailing edge of each blade. The computational fluid dynamics (CFD)-based modelling and simulation of the AFM process can be particularly useful, which can predict the material removal along the blade profile. It can greatly reduce the amount of iterative metrology measurement and testing through the process parameters optimisation, as the CFD-based simulation can be an invaluable aid for the design of the rather complex tooling and optimising metrology operations on a dozen of sections of each blade at the IBR product.

In previous work on the AFM simulation development, the most is focused on the CFD simulation of the process and the abrasive behaviour of the media. Jain et al. utilised a 2D CFD model and simulation that assumed constant media viscosity at 543.48 Pa.s from an unidentified source [1]. The simulation was used to determine the workpiece surface stresses in the AFM process and then use them as inputs for the abrasion model. The simulation model is presented by a purely geometrical assumption, where material removal occurred whenever an assumed spherical simulated grain contacting the workpiece. The simulation was able to predict the trend of the material removal and change in surface roughness with variations of the extrusion pressure, grit size and concentration, and workpiece hardness. The doctoral work by Howard simulated the abrasive media flow using a CFD model which considers the shear rate and temperature dependence of the viscosity [2]. The simulation results are compared with experimental machining results at the point of interest to determine a model correlating with the flow fields [2]. In reference [3], the authors developed a Maxwell material model which should be capable of describing the viscoelastic characteristics of the AFM media. In this published journal article, the flow around a simplified model of an IBR was simulated and tested using the CFD simulation.

However, the modelling and simulation of the AFM processes as developed have some limitations, and many challenges need to be addressed particularly on the prediction and control of the surface generation and topographical profile of the component. One of the challenges is the characterisation of the abrasive media and their complex dynamic behaviour, known as viscoelasticity in the process. The other one is how to monitor and control the surface texture in the surface generation process [4]. The tribology modelling method for the abrasive fluid media and the process are required for developing the scientific understanding of the AFM process both qualitatively and quantitatively, which is essentially important in developing the simulation of AFM processes.

In this paper, the challenges above are addressed by using the following methodologies and further discussed in details. The micro-cutting mechanics modelling intergraded with the Monte Carlo (MC) algorithms/simulation is presented, which attempts to obtain scientific understanding of the grain particle interacting the workpiece surface within the intrinsic AFM process. The integrated modelling and simulation are developed in COMSOL-MATLAB integrated computational environment including its CFD and MATLAB user-defined modules. With the simulation, the surface roughness, topographical profile and the material removal of the blade component can be predicted, which are further evaluated and validated by using real production data provided by industrial partners. The research presented is an essential part of efforts to develop a simulation-based virtual AFM system.

\section{Micro-cutting mechanics modelling and analysis}

\subsection{Methodology for the modelling and simulation}

In abrasive flow machining, it is also essential to simulate the surface generation. In the multiscale model, it can be divided in two parts; one is the CFD module and the other one is the micro-cutting mechanics modelling. The CFD module can simulate the media flow and provide the required specification for the micro-cutting mechanics model. The micro-cutting mechanics model is built on the mechanics for one grain and with the help of the Monte Carlo algorithm, the simulation can be accumulated to mass of grains in the flow. With this combination, the surface generation can be predicted by the simulation program.

In the first part, multiphysics simulation based on fluid structure interaction (FSI) can be used to simulate the working environment configuration of viscoelastic fluid. As a readily available multiphysics simulation tool integrated with CFD capability, COMSOL is used in this research to analyse the AFM media flow in an arbitrary geometry. The CFD modules in COMSOL make it possible to simulate the pressure distribution and shear rate on the surface of the IBR blade. With the appropriate setup of the simulation, the results of AFM process are produced and analysed in the controllable deviation. With previous work, the prediction of material removal and profile accuracy can be predicted with this simulation; it is possible to involve the mechanical model and predict the 
generation process of surface texture and predict the surface roughness.

In the next part, mechanical modelling will be introduced and involved in this simulation to predict the surface roughness and profile accuracy of AFM. It is necessary to build a user-defined function in COMSOL to provide the simulation in micro-scale which makes the prediction of surface roughness possible. The MATLAB function in COMSOL is helpful to involve this micro-mechanical physic model in. In this project the micro-cutting mechanics model is built on the mechanics for one grain and with the help of the Monte Carlo algorithm the simulation can be accumulated to mass of grains in the flow. With this combination, the surface generation can be predicted by the simulation program.

As illustrated in Fig. 1, the simulation-based system is developed and evolved into an industrial feasible virtual AFM system for the process optimisation and the prediction and control of the blade profile accuracy and surface finishing. The criteria for developing the AFM process simulation are in light of the thorough integration of the real process application, physical model, mathematical model and the computational simulation outputs including both graphical and numerical ones. Currently, this project is still on-going with the prediction of surface roughness and surface texture as one of the ultimate goals of the simulation-based virtual machining system development.

\subsection{CFD-based modelling}

From the introduction in the first part, it is necessary to build a CFD modelling environment to simulate the essential inputs for the mechanical modelling, such as distribution of abrasive grains, initial forces and so on. The modelling and simulation is based on multiscale modelling technique combining with multiphysics analysis by taking account of the AFM process nature and industrial production requirements of IBRs from the industrial partner. As a readily available multiphysics simulation tool integrated with CFD capability, COMSOL is used in this research to analyse the AFM media flow in an arbitrary geometry. The CFD modules in COMSOL make it possible to simulate the pressure distribution and shear rate on the surface of the IBR blade. With the appropriate setup of the simulation, the results of AFM process are produced and analysed in the controllable deviation. As illustrated in Fig. 1 , the simulation-based system is developed and evolved into an industrial feasible virtual AFM system for the process optimisation and the prediction and control of the blade profile accuracy and surface finishing.

The CFD module computes multiple variations of the Navier-Stokes equations to model flows in all velocity regimes. This module in the simulation is used to model fluid flow numerically and solve the Navier-Stokes equations, with variations on the simplifying assumptions and constitutive models used for the internal stress related component.

Viscoelastic fluids can be modelled by more complex constitutive equations which consider the elastic time-dependent response of the media. The Oldroyd-B model is a constitutive model used to describe the flow of viscoelastic fluids. The models available can be of the Oldroyd-B type which follow a differential equation that augments the stress term shown in the Navier-Stokes equations described above so that:

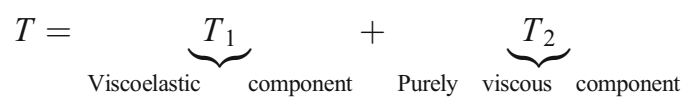

\subsection{Micro-cutting mechanics modelling enhanced by MC method}

It is necessary to find a physical modelling method to simulate the grinding process across the surface. The Hertz theory is widely applied in the grinding process for prediction of indentation depth [5]. As the AFM process is in many ways like to the grinding process, the Hertz theory can be
Fig. 1 Architecture of the virtual AFM system and the associated development

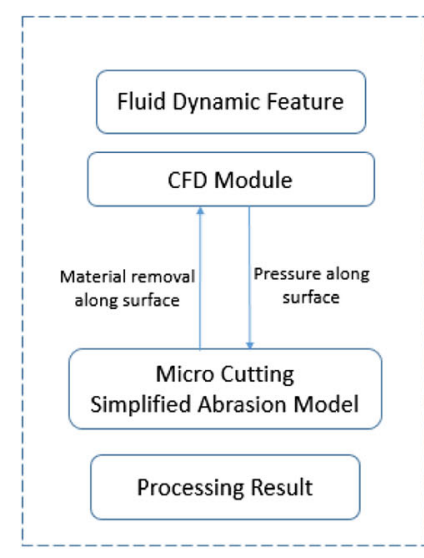

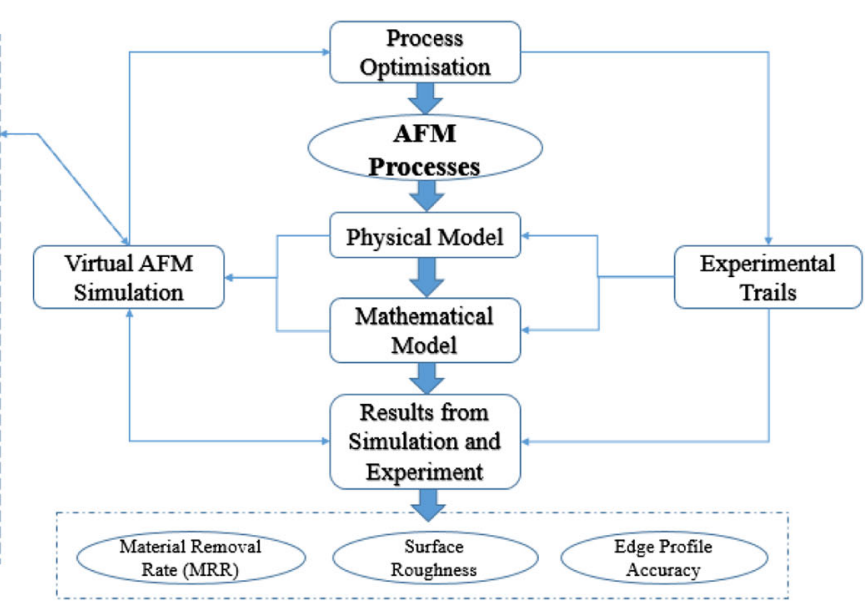


applied in the AFM process for obtaining similar estimation [6]. According to Hertz, the contact area for elastic contact between sphere of radius and a flat surface can be evaluated from:

$\bar{\sigma}=0.41 \times \sqrt[3]{\frac{F_{\mathrm{ng}}^{\prime} E_{m}^{2}}{4 R^{2}}}$

$E_{m}$ denotes Young's modulus of elasticity of the work piece surface and $R$ denotes the spherical radius. $F_{\text {ng }}^{\prime}$ represents the normal force acting on the cutting tool. With this theory, the depth of indentation for elastic loading is given by:

$d^{\prime}=1.55 \times \sqrt[3]{\frac{F_{\mathrm{ng}}^{\prime 2}}{2 R E_{m}^{2}}}$

In this equation, the depth of cutting by each grain can be solved numerically. But it is still not clear which type of cutting it is in current situation. The maximum value of stress at contact area will be equal during plastic deformation will be equal to the Brinell Hardness of the material; thus, the following expression is derived:

$\frac{d^{\prime}}{2 R}=9.22\left[\frac{H_{W}}{E_{m}}\right]^{2}$

This expression is known as the criteria for determining different interaction regimes between the work piece and the abrasive grains; three regimes were identified:

- Chip forming regime: $\frac{d^{\prime}}{2 R}>0.029$

- Plastic regime: $9.22\left[\frac{H_{W}}{E_{m}}\right]^{2}<\frac{d^{\prime}}{2 R}<0.029$

- Elastic regime: $\frac{d^{\prime}}{2 R}<9.22\left[\frac{H_{W}}{E_{m}}\right]^{2}$

The criteria show that when the penetration depth is below $6 \%$ of the grain radius, the chip will not form and the depth is below $0.058 R$, then the displaced material will form ridges by undergoing plastic deformation and no metal will be removed. If the depth is less than the third equation, the grains will slide across the surface and no plastic deformation will occur. With this rule applied to each grain in AFM process, it is possible to simulate the manufacturing across microscale which contributes to surface roughness and the generation of surface texture.

\section{Material removal and surface generation in abrasive flow machining}

\subsection{Modelling of material removal}

Abrasive flow machining is applied by scratching action with abrasive grains in the high viscosity fluid media. As discussed in part two, the normal force produced by the pressure of media will applied to a spherical grain which cause the grain to penetrate in the surface. After this penetration, a groove on the workpiece surface will be produced by grains and the profile of the grain will be produced on the surface. With the grain translated horizontally or even deeper, it will remove material from the workpiece. An idealised classical model of abrasive wear brought out by Jain [1] provides a theoretical basis for the prediction of material removal by AFM process. Jain also evaluated it by accumulating the number of active grains; the shape and depth of the groove produced can be contributed to the volume of stock removal. It makes the material removal of AFM calculated and simulated roughly.

The assumptions adopted to this modelling of material removal by AFM process considered all the grains as the same shape and the load on each particle is constant and equal to the average load [1]. It means in Jain's model, the difference in grains and the influence from the sharpness of each grain are not considered to cut the cost of calculation of simulation and provide a rough prediction of material removal by AFM process. The volumetric material removal in AFM is given as following in Jain's paper:

$V_{i}=2 \pi N l_{s} \frac{R_{c}^{2}}{R_{w}}\left[\frac{d_{g}^{2}}{4} \sin ^{-1} \frac{2 \sqrt{t\left(d_{g}-t\right)}}{d_{g}}-\sqrt{t\left(d_{g}-t\right)}\left(\frac{d_{g}}{2}-t\right)\right] L_{i}$

In this equation, $N$ represents the number of abrasive grains simultaneously acting per unit area of contact. $l_{s}$ is stroke length, $R_{c}$ is radius of media cylinder, $R_{w}$ is radius of cylindrical workpiece, $d_{g}$ is diameter of a spherical grain in this model, $t$ represents the depth of indentation and $L_{i}$ is the length of contact of grain with workpiece surface.

This equation did not consider the difference in grains and the irregular roundness of grains which can only predict the material removal in macro-scale. In the micro-scale, each grain should be considered in statistical methodology so that not only the accuracy of material removal but also the surface texture and profile accuracy can be predicted in better way. In this new type of model, the difference of grain will be involved, and the statistical model will be applied to make the surface closer to the real texture generated by AFM process. 
Fig. 2 Visual representation of the shape factor

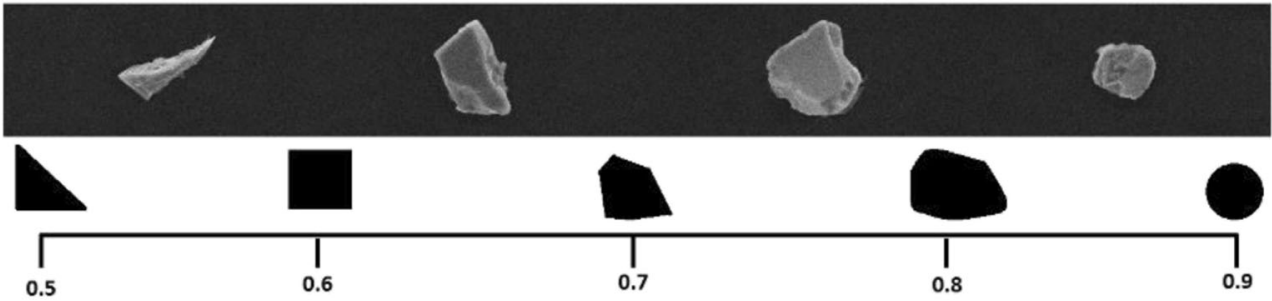

\subsection{The evolution and regeneration of grains in the AFM process}

In the first step, it is necessary to identify the difference between each type of grains. The properties of solid particles such as size, shape, density and hardness have significant influence on slurry erosive wear. To have better understanding of the influence of these individual parameters, the effect of each particle property on erosion needs to be studied separately. There has been some work carried out by other researchers to measure the effect of individual properties on the particle/media wear rate, which includes particle shape, size, particle hardness, density and consistency. The shape of the erosive particles is one of the parameters that affect erosive wear, but its effects are difficult to quantify.

The system to measure the shape of the particles is to study the shape factor of them. Desale showed the effect of shape of particles on erosive wear in their experimental work [7]. They selected three different materials with the same size as erodent particles and determined their shape factor (SF) using the following relationship:

$\mathrm{SF}=\frac{4 \pi A}{P^{2}}$

In this definition, $A$ is the projected area $\left(\mu \mathrm{m}^{2}\right)$ and $P$ is the overall perimeter $(\mu \mathrm{m})$ of the projection of a solid particle.
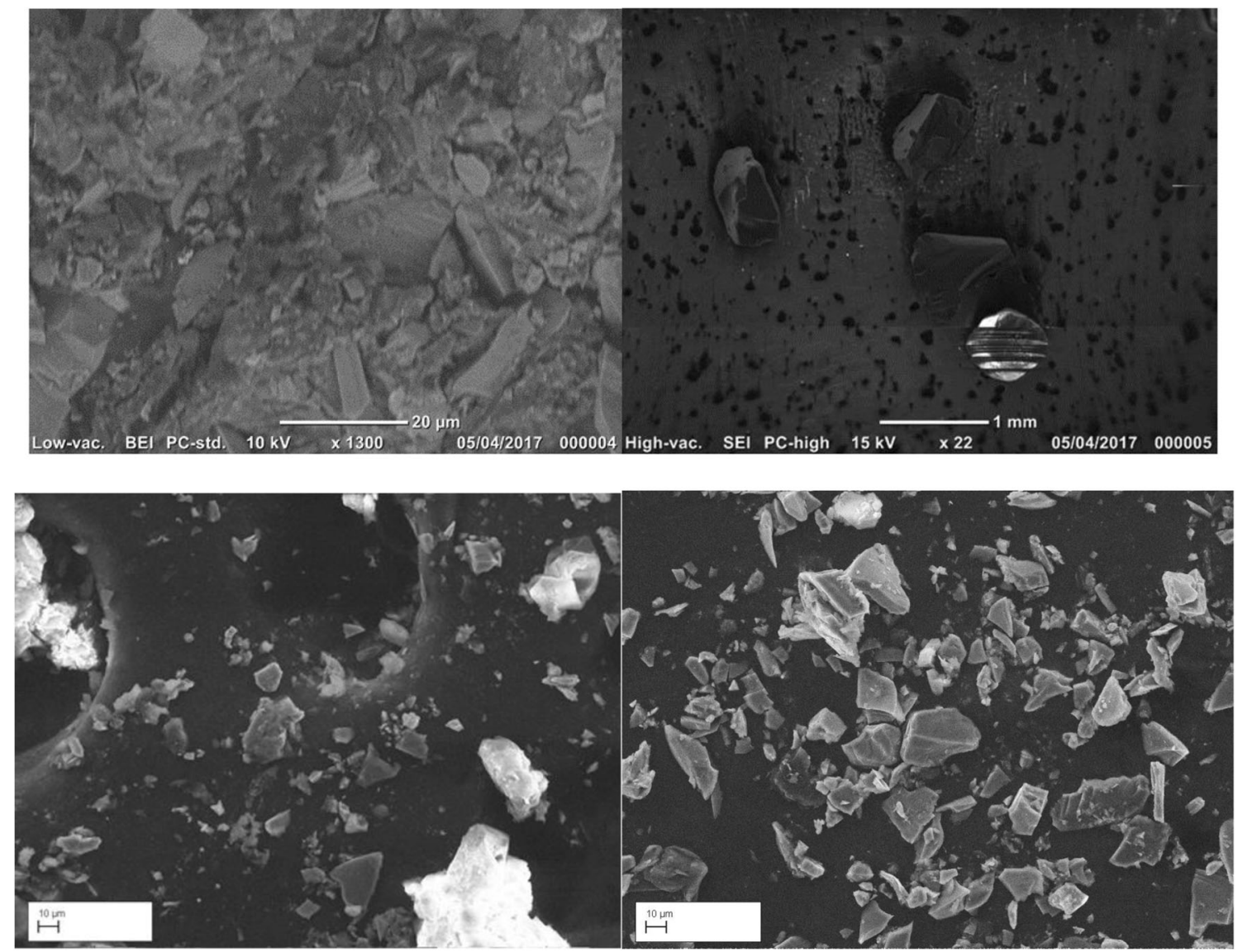

Fig. 3 Image of the sample under microscope 
Fig. 4 Grain particle variations in the 'random' surface generation process
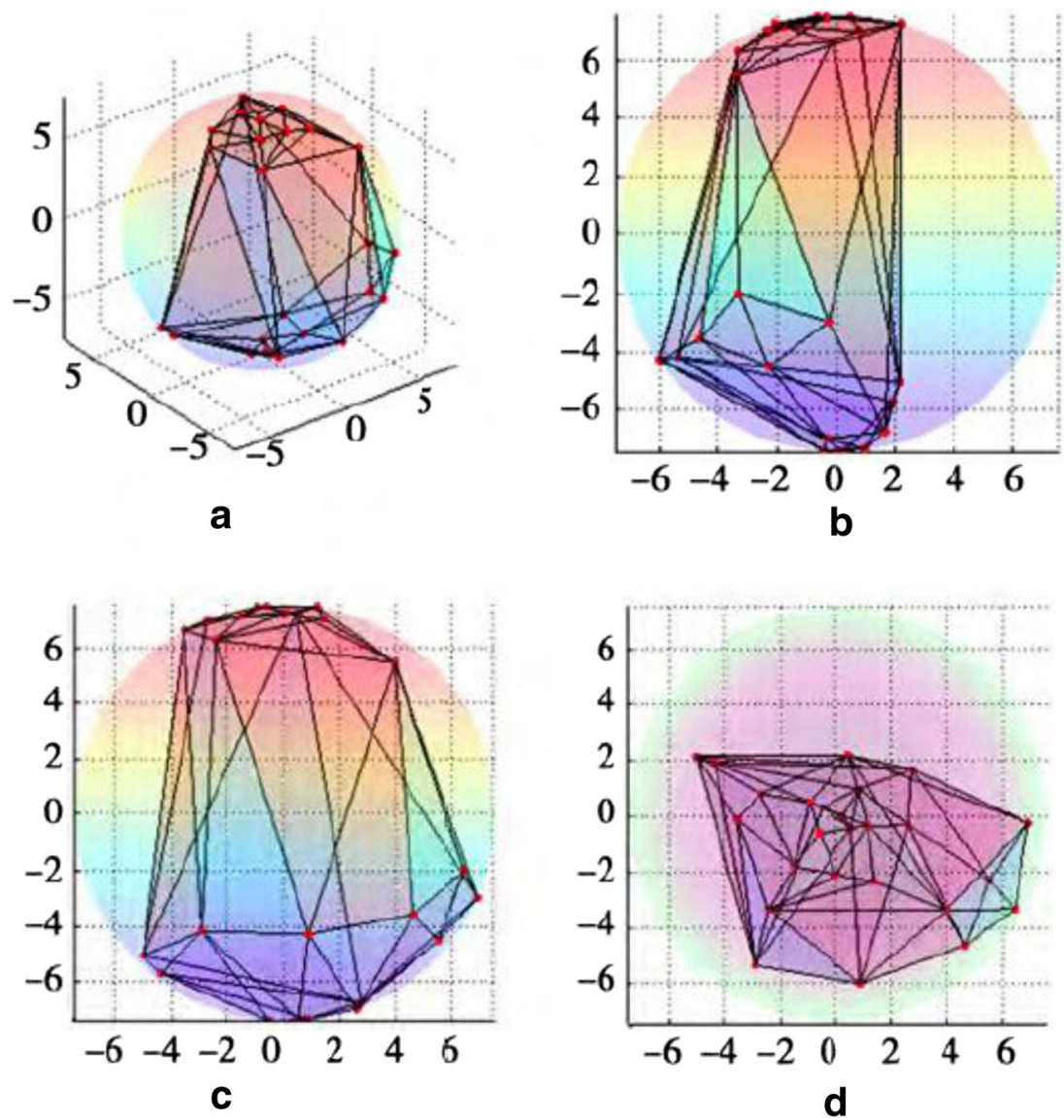

The shape factor decreased with increase in angularity of the particles. The example of this factor is shown in Fig. 2.

With the knowledge of shape factor, it is possible to generate random grain in different shapes to make the prediction more particular and meticulous. In this model, ODEC method brought out by Kruggel-Emden was adapted and be applied to 3D [8]. In this method, the grains will be generated randomly in sphere with specific radius in following steps. At first, a number of points will be generated at the surface of the sphere. After the generation of these points a well-shaped graph will be created and tested to get the shape factor of this grain. If the grain does not meet the required shape factor, some more points at the surface will be added into this graph. After a few times of this step, the grain will have the same shape factor with the data from real grains.

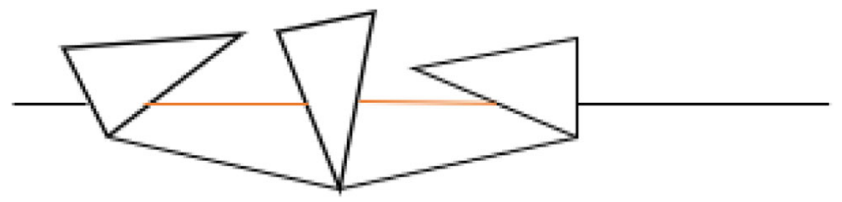

Fig. 5 The cutting path of one grain (grey line is the state before machining)
In Kruggel-Emden's paper, they only adapt this method to 2D situation; it is necessary to involve it into the 3D environment. In 3D situation, the shape factor will be taken from three views: front view, top view and side view. In this model, the grain generated randomly will be test in these three views and calculated by following formula:

Shape factor $=\frac{A_{\text {front }}+A_{\text {side }}+A_{\text {top }}}{C_{\text {front }} A_{\text {front }}+C_{\text {side }} A_{\text {side }}+C_{\text {top }} A_{\text {top }}}$

In this equation, $C$ represents the shape factor of projection from grain in each view while $A$ represents the area of the projection from grain in each view. The sample grains from fluid media under microscope are shown in Fig. 3. The results of the generation and the

Table 1 Collection of data from the grains measured in this project

\begin{tabular}{llll}
\hline & Variance & Mean & Standard deviation \\
\hline Perimeter & 1337.17143 & 42.3424356 & 36.5673547 \\
Shape factor & 0.01612654 & 0.71243735 & 0.12699033 \\
$W / L$ aspect ratio & 0.0260009 & 0.65829813 & 0.16124796 \\
\hline
\end{tabular}




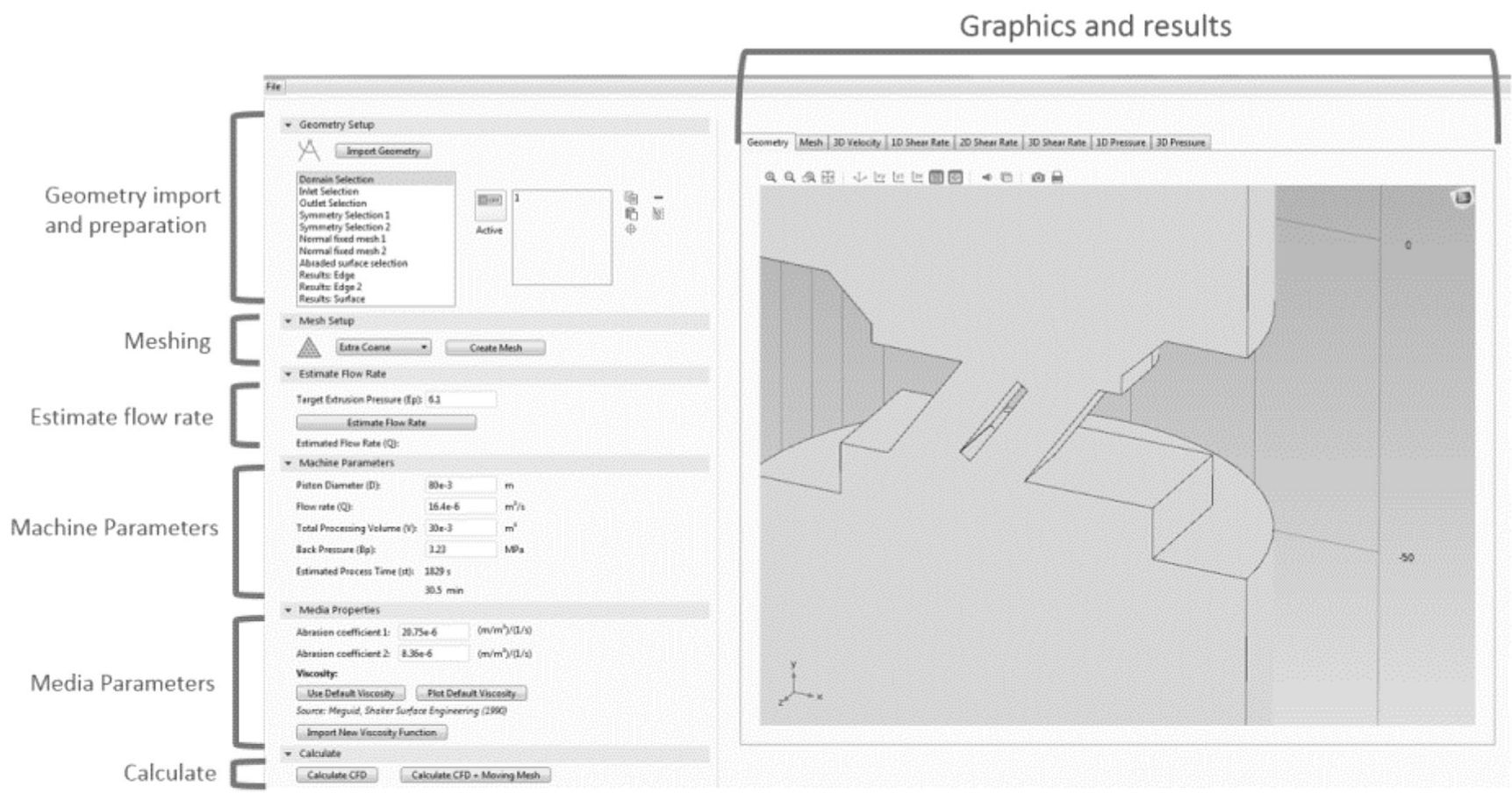

Fig. 6 Interface of the simulation

projection of the grain are shown in Fig. 4 in the 'random' surface generation process.

The generation code in MATLAB helps COMSOL to build the exact shape for the particles in simulation process and helps to predict the surface generation process during the AFM process. The generation of grains also provides the possible for mechanism model mentioned below to be calculated during the AFM process to predict the surface generation in micro-scale.

\subsection{The locus equation representing the machining process by one single grain}

After the generation of grains, it is possible to predict the material removal in micro-scale. With the help of Eq. (3), it is possible to get the cutting depth of each grain. Different from Sallam's research, the building of random grain will make the depth of cutting changing all the time while the grain is rolling on the workpiece surface. As described before, the grains are

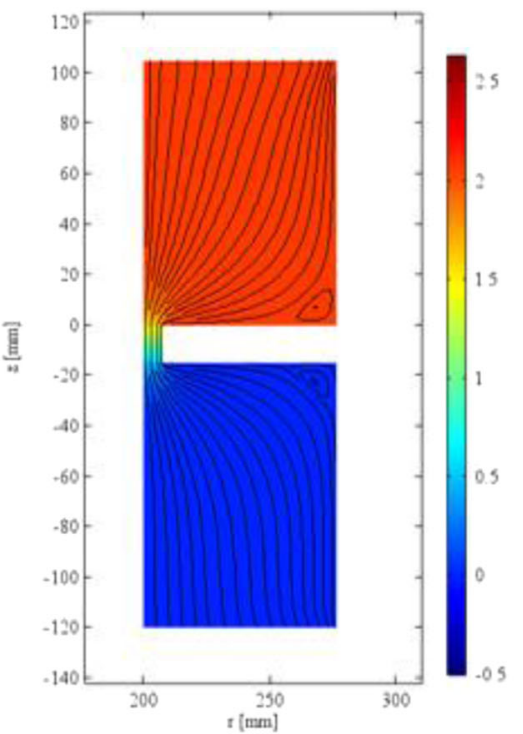

a

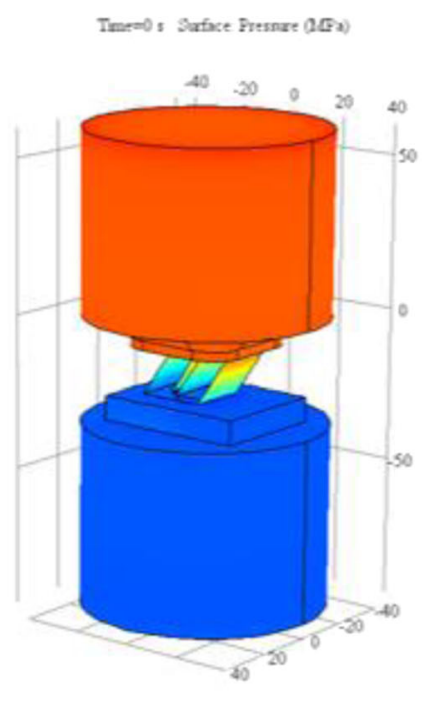

b

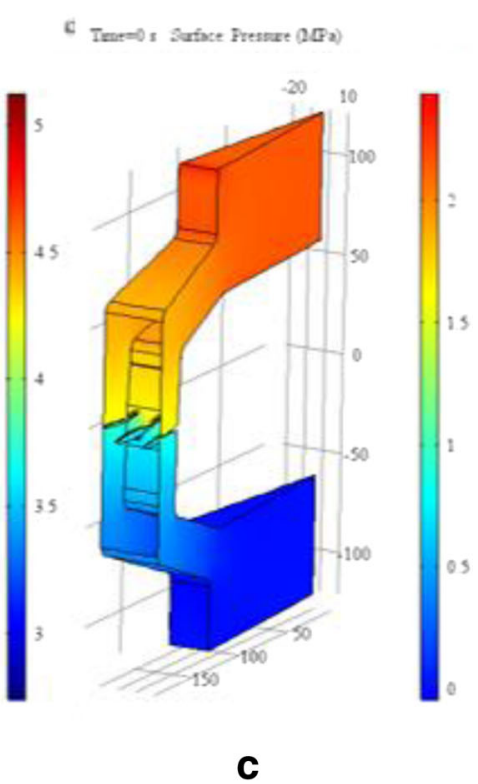

Fig. 7 a Davies (1993) dissertation case; b Extrude Hone trails; c ITP IBR study cases 
Table 2 Simulation test based on experimental cases on different geometries

\begin{tabular}{lll}
\hline Geometry & Simulated Pressure drop (MPa) & Experimental Pressure drop (MPa) \\
\hline Davies (1993) dissertation & 2.07 & 2.24 \\
Extrude Hone trial & 1.67 & 1.77 \\
ITP IBR study case & 1.63 & 1.59 \\
\hline
\end{tabular}

carried by fluid with high pressure and flowing and polishing along the surface. It means the grains will roll along the surface and leave the cutting pattern according to the shape of each grain. The depth of cutting will increase while the grain is rolling towards its sharper side and the cutting depth will decrease while it is rolling to the other side. In Fig. 5, a simple triangle grain is shown as an example of this model.

In Eq. (3), the depth is mainly decided by the normal load on the grain and the average radius of grain. In Sallam's model, the load on the grain will not change at all time, which keeps the depth the same during the entire process. In physical model, the load is determined by the pressure along the surface and the contact area of the grain and workpiece surface as following equation:

$F=P \cdot A$

In this equation, $P$ represents the pressure along the surface while $A$ represents the contact area of the grain and workpiece. If we assume that the radius of grain is constant while rolling along the surface, we can get the equation with depth and contact area with Eqs. (3) and (7) as follows:

$d=1.550 \sqrt[3]{\frac{P^{2} \cdot A^{2}}{2 R E_{m}^{2}}}$
Equation (9) provides a method to calculate the depth of cutting while the grain is rolling along the surface in the entire process and generate a curve to simulate the locus equation of machining process by single grain. With this model, the material removal and surface texture can be calculated and predicted in micro-scale.

\subsection{The Monte Carlo method applied to AFM modelling}

Abrasive flow machining process is a type of stochastic process. Not only the shape of grains and the size distribution of the grains in fluid media but also the collision positions and angles by grains are in different scales of variation. It has been realised by many researchers that the Monte Carlo simulation is a practical tool to predict the wear rate of materials. In abrasive flow machining, the Monte Carlo simulation shows some light in providing a method to generate several random generated grains and make the grains cutting at any place along the workpiece surface with a random speed and direction that is possible. With enough machining process of grains and correct distribution of machining position and direction of each grain, the Monte Carlo simulation will provide a clear and accurate result on the generation of surface texture. In the meantime, the
Fig. 8 Flow chart of microcutting mechanics modelling and analysis

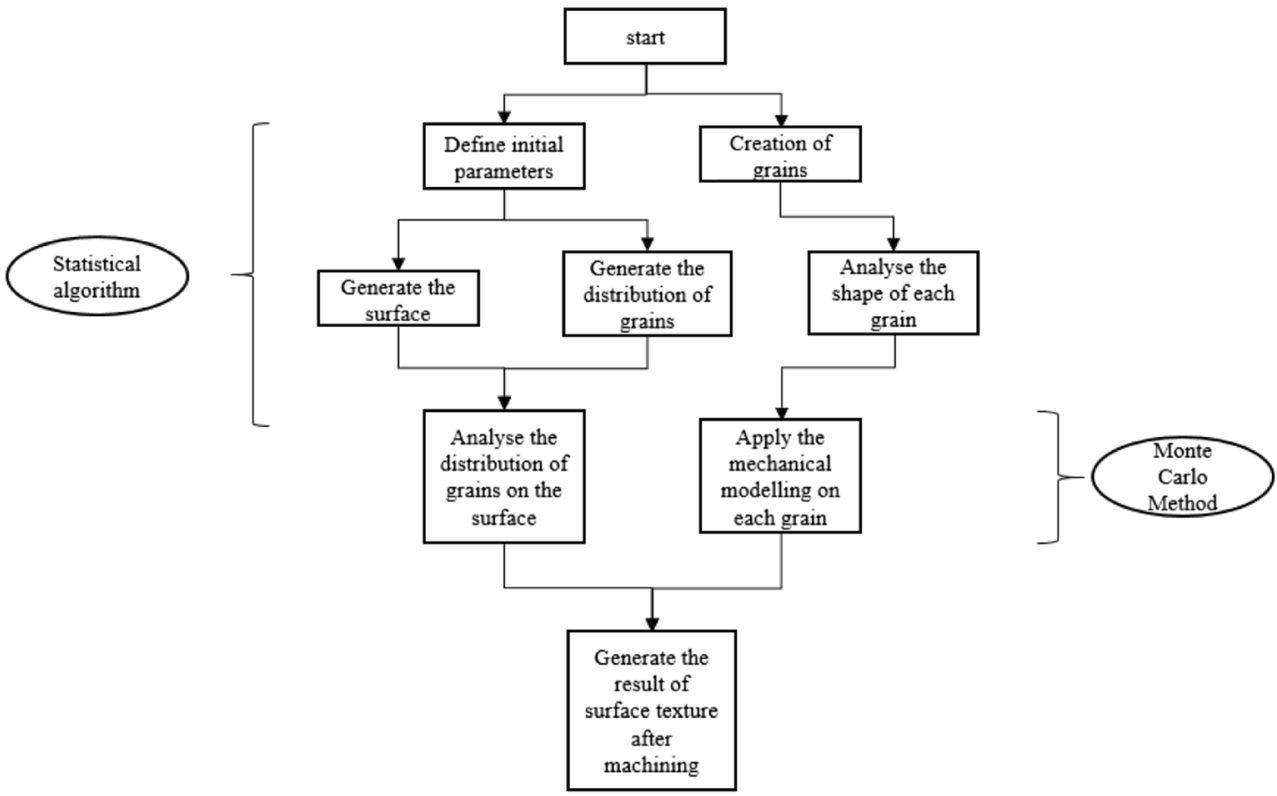




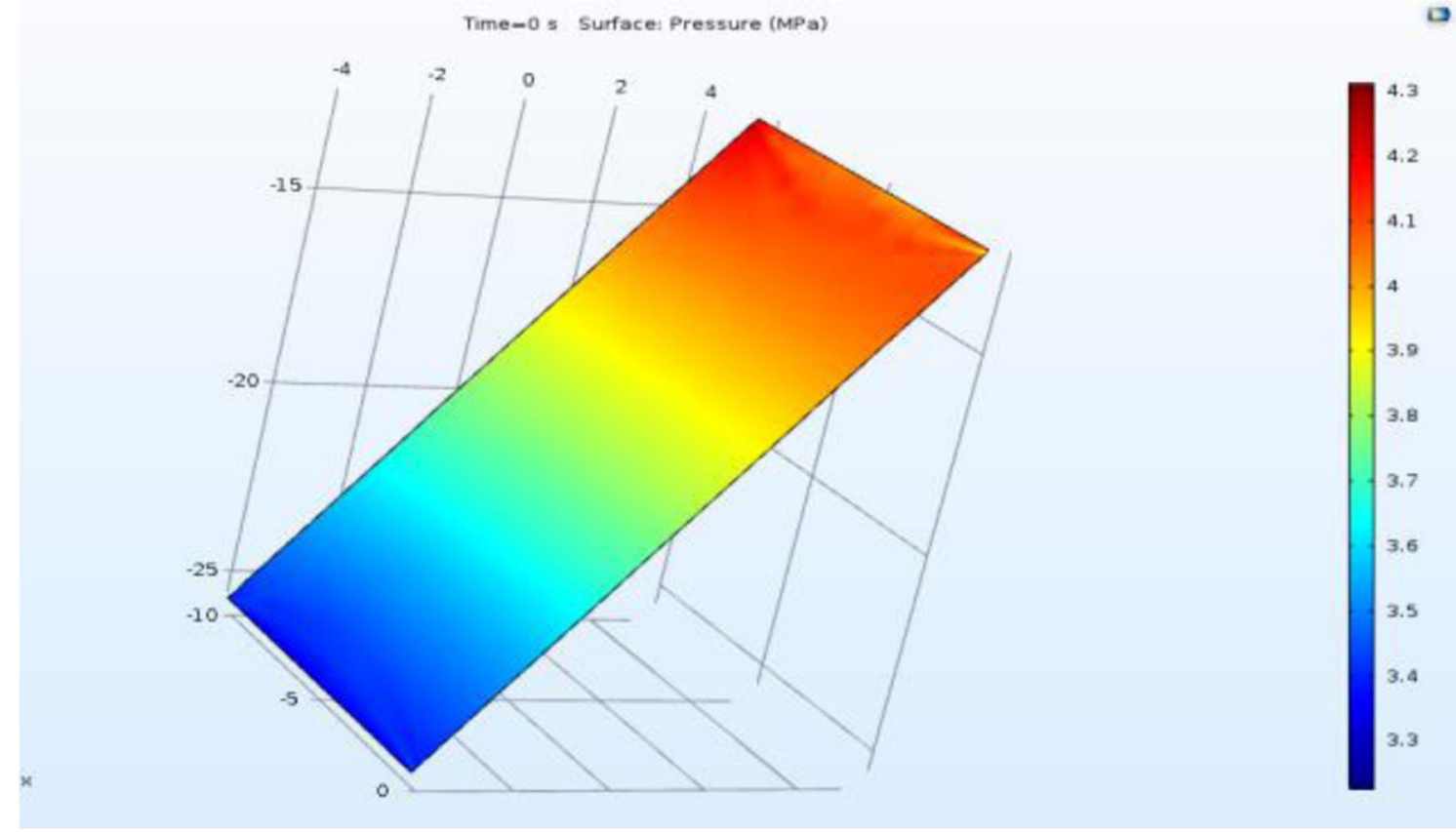

Fig. 9 Pressure distribution of the fluid media on workpiece surface

prediction of material removal and surface roughness can also be provided in higher accuracy.

First, it is necessary to gather the information about the grains. The grains in AFM are almost all in the different shapes and the sharp factor of them is involved in a large distribution. In this project, all the experiment is based on the media provided by Extrude Hone; the collection of data in Table 1 also comes from the fluid media with the code named as $649-\mathrm{Z}$ produced by Extrude Hone.
With these data, a set of grains are generated with the normal distribution on the Monte Carlo method in MATLAB to provide the calculation of mechanism modelling code. The mechanism modelling simulation will based on these randomly generated grains and will be more accurate with the result.

On the other hand, it is also important to find out a way to put these grains into the fluid and let them start the machining along the workpiece surface. In this model, the distribution of machining position is the uniform distribution in the

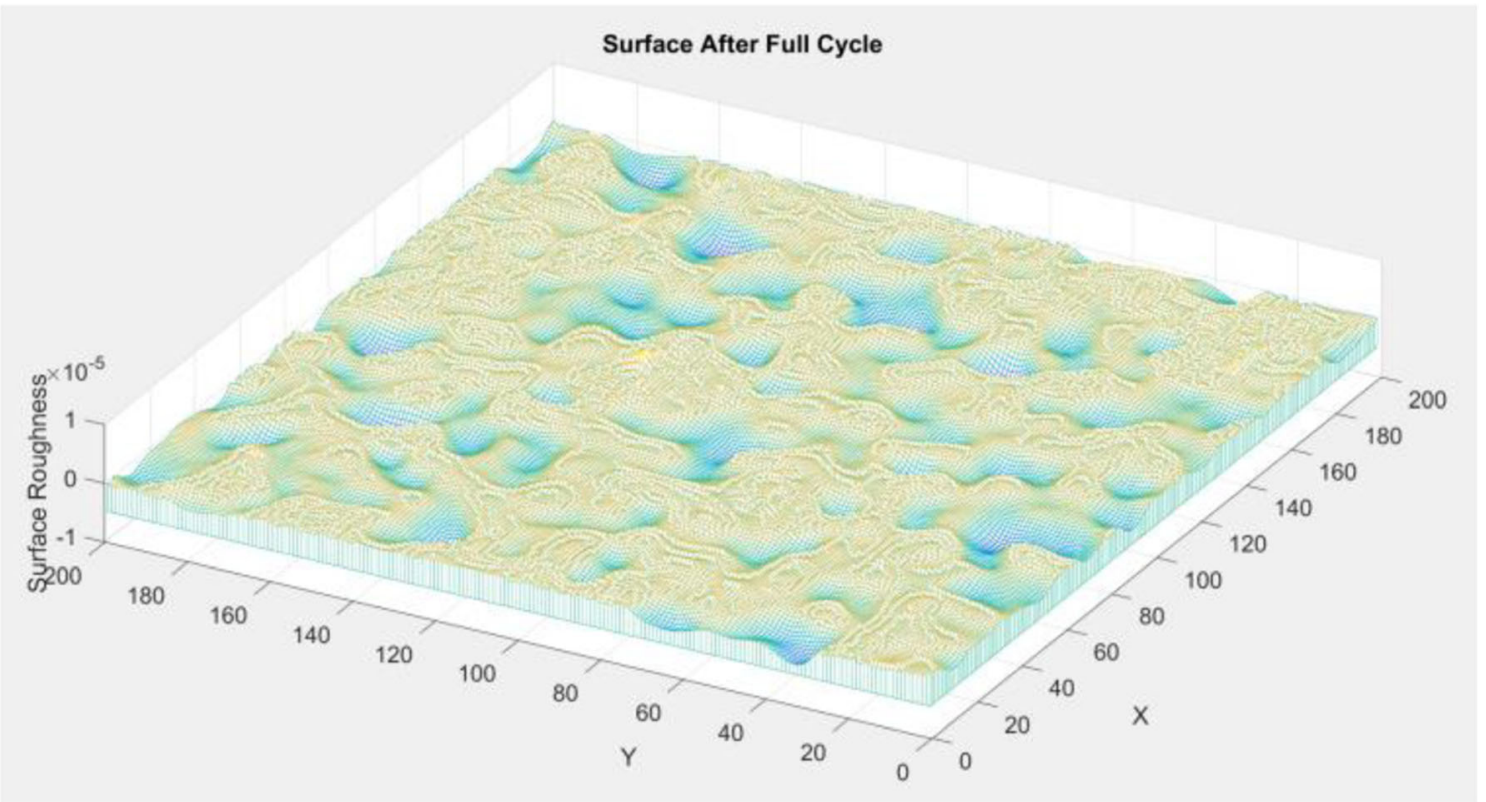

Fig. 10 The prediction of surface roughness after first cycle 
Fig. 11 A 3D CAD model of AFM trial fixture; test coupon shown, blue; hardened steel coupon clamp, red

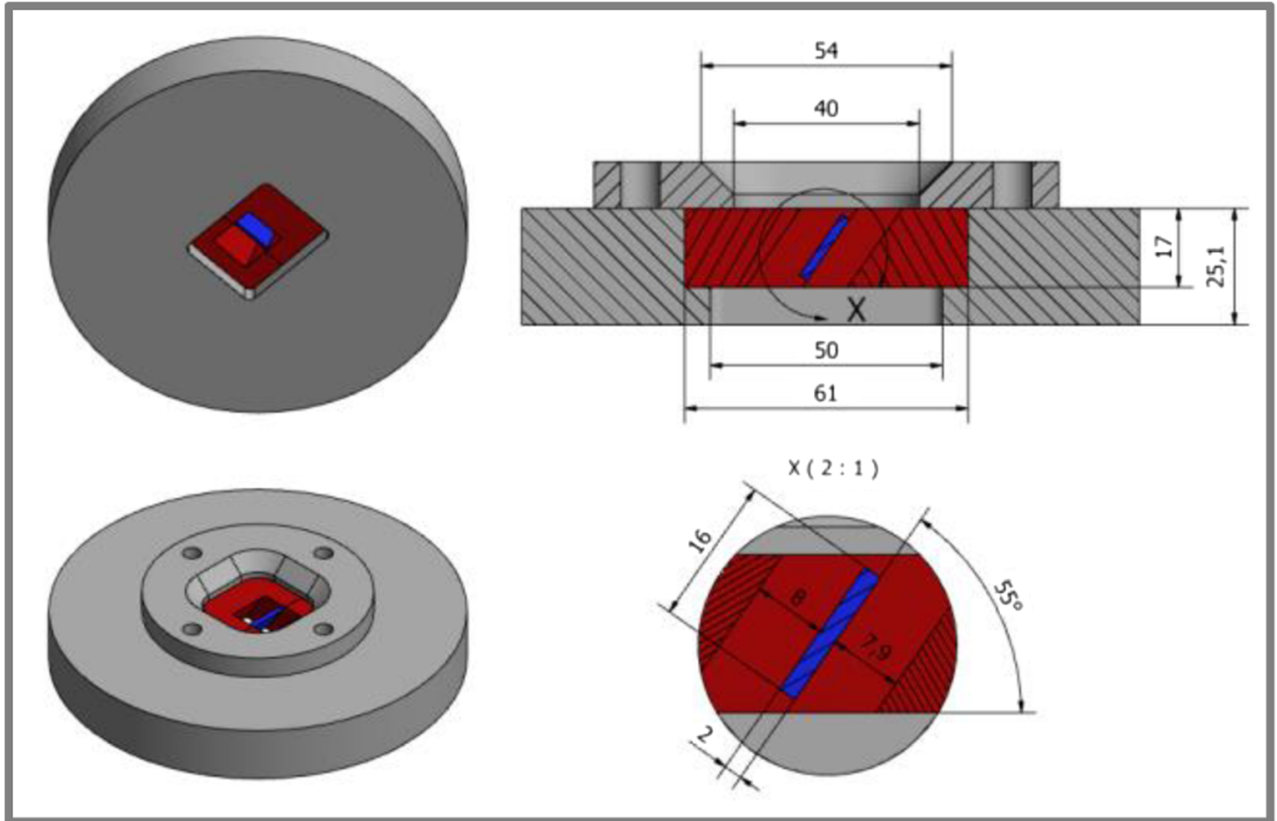

fluid media while the distribution type of the direction and speed is the Gaussian distribution with the direction and speed of fluid media. With the help of the Monte Carlo method, the grains are put into the fluid media with uniform distribution as the possibility of grains appearing at any position is equal. The direction of each grain will be mostly the same with the direction of fluid media as the viscosity of the media is very high. The grains will stay almost the same method of moving with the fluid. Considering the little deviation of each grain, the direction and speed of each grain are added with a small noise with the Gaussian distribution. After these setup, a number of grains will be calculated in mechanism model the result of material removal by each grain will be added up and contributes to a final result of material removal and surface texture of the workpiece surface. In this MATLAB code, the simple surface generation will be simulated based on the results from CFD module simulation in COMSOL and the result will also be stored and import back to COMSOL simulation which make the simulation as an entirety.
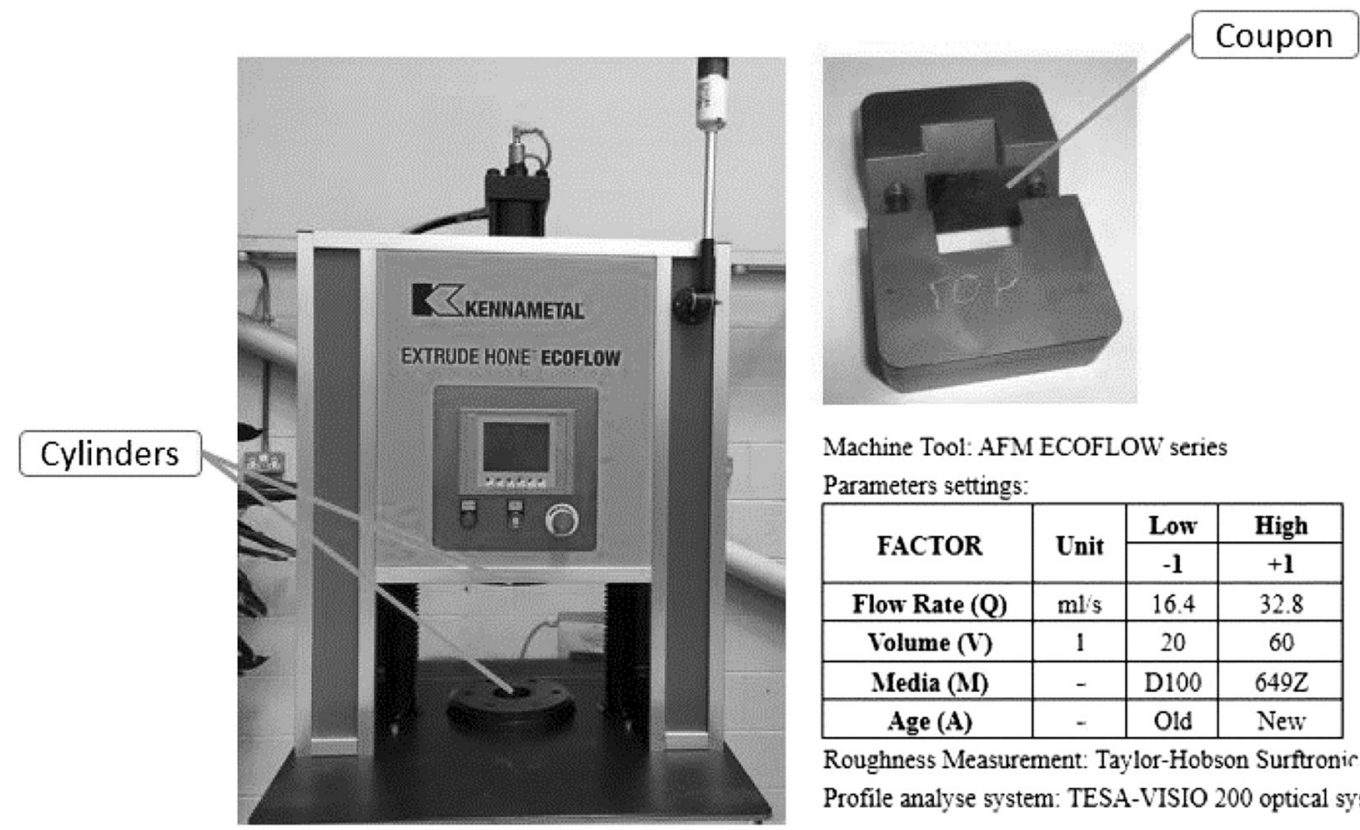

Machine Tool: AFM ECOFLOW series

Parameters settings:

\begin{tabular}{|c|c|c|c|}
\hline \multirow{2}{*}{ FACTOR } & \multirow{2}{*}{ Unit } & Low & High \\
\cline { 3 - 4 } & & $-\mathbf{1}$ & $+\mathbf{1}$ \\
\hline Flow Rate (Q) & $\mathrm{ml} / \mathrm{s}$ & 16.4 & 32.8 \\
\hline Volume (V) & 1 & 20 & 60 \\
\hline Media (M) & - & D100 & $649 \mathrm{Z}$ \\
\hline Age (A) & - & Old & New \\
\hline
\end{tabular}

Roughness Measurement: Taylor-Hobson Surftronic S-100 stylus Profile analyse system: TESA-VISIO 200 optical system

Fig. 12 The AFM experimental trial setup 
Table 3 AFM trial levels

\begin{tabular}{llll}
\hline FACTOR & Unit & $\begin{array}{l}\text { Low } \\
-1\end{array}$ & $\begin{array}{l}\text { High } \\
+1\end{array}$ \\
\hline Flow rate $(Q)$ & $\mathrm{ml} / \mathrm{s}$ & 16.4 & 32.8 \\
Volume $(V)$ & 1 & $20(10)$ & $60(30)$ \\
Media $(M)$ & - & D100 & $649 \mathrm{Z}$ \\
Age $(A)$ & - & Old & New \\
\hline
\end{tabular}

\section{Simulation and experimental verification}

\subsection{Multiphysics simulation with CFD modules within COMSOL}

The simulation based on CFD module within COMSOL is aimed to provide the prediction of material removal in this research. However, it is also useful to provide the initial force and pressure for mechanical model on grinding along the surface.

In this simulation program, the accuracy of pressure distribution and velocity distribution along the surface is important. These two key factors influence a lot in the prediction of surface texture so it is important to calculate it in CFD model to generate the distribution along each surface accurately. Figure 6 shows the simulation interface, which interactively provides the prediction of the pressure distribution under any IBR configuration. With this simulation, three different industrial application cases are applied and thoroughly tested and further validated with the experimental results.

In Fig. 7, the simulations were set up with the flow rate measured in the experiments and the simulated pressure drop was obtained from the average at the inlet face minus the average at the outlet; in the case of backpressure-enabled experiments, the value was set at the outlet boundary condition; the results are summarised in Table 2. The first test is based on Davies' experiment [9] while cases 2 and 3 are recorded in reference [3].

\subsection{User-defined MATLAB codes in COMSOL for micro-cutting mechanics analysis}

The multiphysics simulation based on COMSOL provides a lot of information for the mechanical modelling which aims to predict the surface roughness and the generation of surface texture [10]. It is possible to write a MATLAB code to enhance the function in COMSOL to provide the prediction of surface roughness. A brief flow chart of this code is shown in Fig. 8 .

The user-defined MATLAB code is mainly built on the contribution of mechanism model and the Monte Carlo method and the source data from the CFD simulation based on COMSOL. The pressure along the surface and velocity of fluid media is simulated by COMSOL and imported into the mechanism model. After the generation of grains in the specific range of sharp factor, the grains are imported into the mechanism model and distributed into random position with initial velocity and pressure from CFD simulation to start the calculation of material removal by each grain. With the help of the Monte Carlo simulation in MATLAB, these grains are applied on the mechanism model simulation and contribute to the machining process by each of them.

In this MATLAB code, the data about pressure and velocity distribution of fluid media along the workpiece surface is imported from the simulation based on COMSOL as described before. The example of pressure distribution of fluid media is shown in Fig. 9. With the help of pressure and velocity, the mechanism model can be applied to the random generated grains built from MATLAB to simulate the generation process of AFM along a small piece of workpiece surface as an example. With one single calculation, one line of wave will be generated with the result of mechanism model which represents the cutting depth through this whole line with one grain. With the help of the Monte Carlo method, the calculation is done times after times randomly and the result will be accumulated in this plane.

\subsection{Simulation for predicting the surface generation}

After the calculation process of MATLAB code, it is necessary to bring the result back to the simulation in COMSOL for further calculation. As the result shown in Fig. 10, the surface texture is somehow shown in MATLAB format. With the LiveLink for MATLAB and COMSOL, these data can be communicated and updated at the same time. With this kind
Table 4 Results of surface roughness according to the experimental trails

\begin{tabular}{llllllll}
\hline $\begin{array}{l}\text { Trail } \\
\text { number }\end{array}$ & \multicolumn{2}{l}{ SR LR (across) } & & \multicolumn{2}{l}{ SR TB (along) } \\
\cline { 2 - 3 } & $\begin{array}{l}\text { I SR LR } \\
(\mu \mathrm{m})\end{array}$ & $\begin{array}{l}\text { F SR LR } \\
(\mu \mathrm{m})\end{array}$ & $\begin{array}{l}\text { IMP SR LR } \\
(\%)\end{array}$ & & $\begin{array}{l}\text { I SR TB } \\
(\mu \mathrm{m})\end{array}$ & $\begin{array}{l}\text { F SR TB } \\
(\mu \mathrm{m})\end{array}$ & $\begin{array}{l}\text { IMP SR TB } \\
(\%)\end{array}$ \\
\hline Run \#1 & 1.54 & 0.88 & 43 & 0.67 & 0.23 & 64.7 \\
Run \#2 & 1.78 & 0.74 & 59 & 0.71 & 0.22 & 67.4 \\
Run \#3 & 1.02 & 0.71 & 30 & 0.25 & 0.21 & 11.4 \\
Run \#4 & 1.30 & 0.49 & 63 & 0.42 & 0.16 & 59.3 \\
\hline
\end{tabular}


Fig. 13 Surface roughness measurement locations and directions

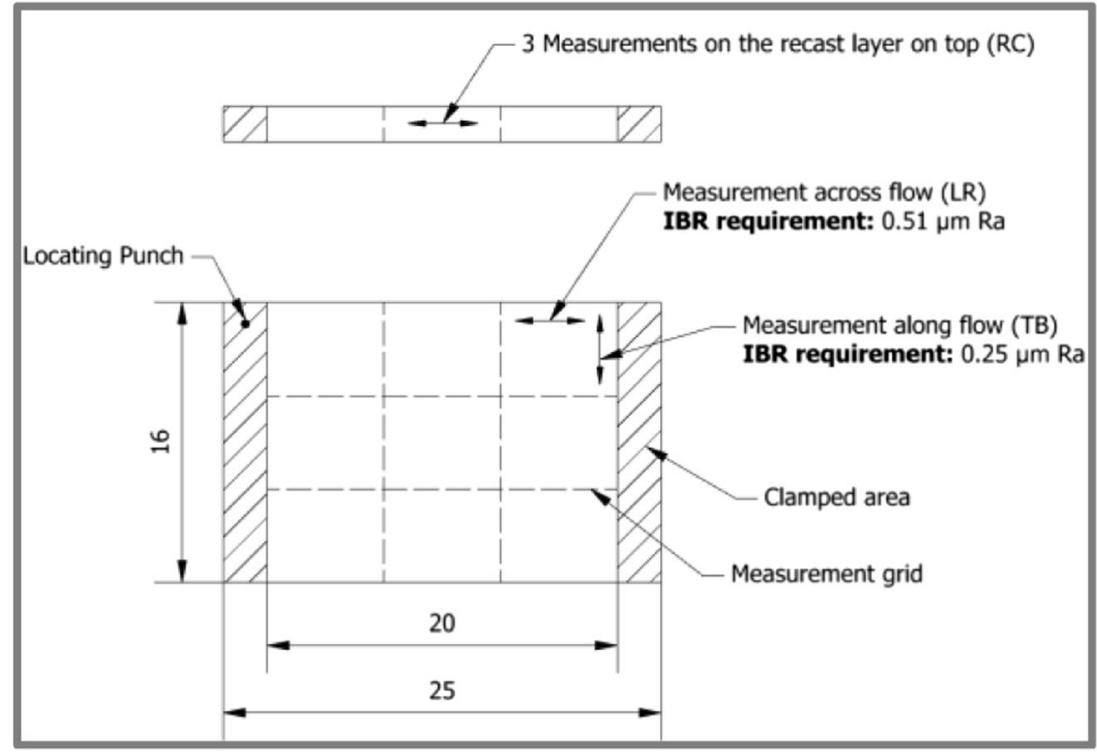

of help, the simulation can stay at a better stage on the prediction accuracy on both surface roughness and material removal.

After the communication, the result of material removal will be sent back to COMSOL and the workpiece will change as the exact result from MATLAB to continue the simulation in the next cycle. It is also possible for COMSOL to read the surface roughness and profile accuracy through the connection between COMSOL and MATLAB which make this simulation more like a complete virtual AFM simulation.

\subsection{Experimental trails and validation on simulation}

An initial set of experiments was conducted during development of this dissertation as part of a NATEP (National Aerospace Technology Exploitation Programme)-funded project, with the goal to measure the material removal and surface roughness under specific configurations. The experiments and additional production data can be used to further develop simulation models.

The design of the experiment was developed with project partners at a seminar conducted by NATEP on the topic; the experiment is a four-factor full factorial design, with two

Table 5 Simulation results of surface roughness based on experimental trail 1

Surface roughness TB (along) $(\mu \mathrm{m})$

Initial Ra value

Ra value after quarter cycle

Ra value after half cycle

Ra value after third quarter cycle

Ra value after full cycle
0.66864

0.55564

0.40497

0.28978

0.21414 levels (high and low) on each factor; the agreed upon input factors are as follows: flow rate, volume, media, media age.

The test piece is made from Inconel 718. Test piece geometry was designed by the project partners to resemble IBR geometry as much as possible within complexity and budget limits; it is shown in Fig. 11 and the setup, detailed process parameters and the coupon in experiments are shown in Fig. 12.

After AFM process, the roughness measurements where performed along two main directions shown in the figure below; reference values for the tolerance that IBR manufacturers have to achieve is also given; it varies depending on the direction of the flow.

The edge profile for the six first coupons tested was also moulded and captured with a TESA-VISIO 200 optical system, with the intent of comparison with the profile obtained by simulation. The high-low levels for the factors are shown in Table 3, while the results and improvement of experimental trails based on the AFM process are shown in Table 4 .

After the experiment, the simulation based on the configuration of these experimental trails has been tested and the results according to material removal and surface roughness have been compared which shows the simulation shows some light on predicting the AFM process both on material removal and surface roughness (Fig. 13) [3]. The results of surface roughness in the simulation according to experimental trail \#1 are shown in Table 5.

\section{Conclusions}

The modelling method and results of simulations have provided the insight on the relative importance of the different AFM process parameters involved. From the analysis of 
experimental trials, it is found the abrasive media $(M)$ and volume $(V)$ consistently create the largest difference in the machining system. Furthermore, the volume $(V)$ as the go-to parameter for tuning a process is exceptionally easy to be modified, while the media $(M)$ is harder to be modified, as the industrial companies using AFM processes for IBR manufacturing will likely require prohibitively large batches for industrial testing before switching media.

The development of the abrasion model provides an effective approach for predicting the surface roughness and profile accuracy of the component in the AFM process particularly by integrating with results from CFD simulation [11-13]. With theoretical and experimental considerations, flow rate and pressure distribution along the workpiece surface have been adopted as main factors in the model. With the further development of micro-cutting mechanics modelling and analysis, it leads to better scientific understanding of the process, development of the prediction models and the process optimisation [14-17].

The AFM modelling and simulation presented are currently under further development with more production data input from industrial partners and shop floor trials, as the part of the efforts in developing the industrial feasible virtual AFM system [18-20].

Acknowledgements The authors are thankful for the regular meetings and discussions with industrial partners at the project consortium.

Funding information This study is financially supported by the National Aerospace Technology Exploitation Program (NATEP) (Project No. MAA073).

Open Access This article is distributed under the terms of the Creative Commons Attribution 4.0 International License (http:// creativecommons.org/licenses/by/4.0/), which permits unrestricted use, distribution, and reproduction in any medium, provided you give appropriate credit to the original author(s) and the source, provide a link to the Creative Commons license, and indicate if changes were made.

\section{References}

1. Jain R, Jain V, Dixit P (1999) Modeling of material removal and surface roughness in abrasive flow machining process. Int J Mach Tool Manu 39:1903-1923, 1999

2. Howard M, Cheng K (2014) An integrated systematic investigation of the process variables on surface generation in abrasive flow machining of titanium alloy 6A14V. Proc IMechE B J Eng Manuf 228(11):1419-1431
3. Cheng K, Shao Y, Bodenhorst R, Jadva M (2017) Modelling and simulation of material removal rates and profile accuracy control in abrasive flow machining of the IBR blade and experimental perspectives. Trans ASME J Manuf Sci Eng 139(12):121020

4. Kumar SS, Hiremath SS (2016) A review on abrasive flow machining (AFM). Procedia Technol 25:1297-1304

5. Rowe WB, Qi HS, Morgan MN, Zheng HW (1993) The real contact length in grinding based on depth of cut and contact deflections. In: Proceedings of the Thirtieth International MATADOR Conference. Palgrave, London, pp 187-193

6. Vogel L, Peukert W (2004) Determination of material properties relevant to grinding by practicable labscale milling tests. Int $\mathrm{J}$ Miner Process 74:S329-S338

7. Cheng K (ed) (2008) Machining dynamics: theory, applications and practices. Springer, London

8. Kruggel-Emden H, Simsek E, Rickelt S, Wirtz S, Scherer V (2007) Review and extension of normal force models for the discrete element method. Powder Technol 171(3):157-173

9. Davies PJ (1993) The rheological and honing characteristics of polyborosiloxane/grit mixtures (Doctoral dissertation, Sheffield Hallam University)

10. Jain R, Jain V (1999) Simulation of surface generated in abrasive flow machining process. Robot Comput Integr Manuf 15:403-412

11. Gorana VK, Jain VK, Lal GK (2006) Forces prediction during material deformation in abrasive flow machining. Wear 260(1-2): 128-139

12. Barnes HA (2003) A review of the rheology of filled viscoelastic systems. Rheology Reviews (The British Society of Rheology, pp $1-36$

13. Wan S, Ang YJ, Lim GC (2014) Process modelling and CFD simulation of two-way abrasive flow machining. Int J Adv Manuf Technol 71(5):1077-1086

14. Malkin S, Guo C (2008) Grinding technology: theory and application of machining with abrasives. Industrial Press Inc

15. Marinescu ID, Rowe WB, Dimitrov B, Inaski I (2004) Tribology of abrasive machining processes. Elsevier

16. Fang L, Cen Q, Sun K, Liu W, Zhang X, Huang Z (2005) FEM computation of groove ridge and Monte Carlo simulation in twobody abrasive wear. Wear 258(1-4):265-274

17. Mooney CZ (1997) Monte Carlo simulation, vol 116. Sage Publications

18. Weinan E (2011) Principles of multiscale modeling. Cambridge University Press

19. Howard M, Cheng K (2013) An industrially feasible approach to process optimization of abrasive flow machining and its implementation perspectives. Proc IMechE B J Eng Manuf 228(11):14191431

20. Desale GR, Gandhi BK, Jain SC (2011) Development of correlations for predicting the slurry erosion of ductile materials. J Tribol 133:031603. https://doi.org/10.1115/1.4004342

Publisher's note Springer Nature remains neutral with regard to jurisdictional claims in published maps and institutional affiliations. 